\title{
A CONTRIBUIÇÃO DA GEOGRAFIA MÉDICA E DA SAÚDE NA PANDEMIA DO COVID 19
}

uma reflexão sobre a cidade do Rio de Janeiro

\author{
Marta Rocha de Castro ${ }^{1}$ \\ Departamento de Geografia- PUC* RJ \\ martarochaacupuntura@gmail.com
}

\begin{abstract}
Resumo
A Geografia da saúde é uma disciplina que faz uso dos conceitos geográficos para trabalhar com os determinantes de saúde e doença e as influências dos aspectos sociais, ambientais, econômicos e culturais na saúde de determinada população. O Texto traz uma reflexão acerca da importância da Geografia da Saúde durante a pandemia do novo coronavírus, especificamente na cidade do Rio de Janeiro, Brasil. Consideramos que todo o processo de pandemia no Brasil foi marcado pelo negacionismo em relação a doença e a ciência, fato esse que agravou o quadro de contaminados e de mortos pela ausência de políticas públicas eficientes que estimulassem medidas preventivas que minimizassem a disseminação da doença
\end{abstract}

Palavras-chave: Geografia da Saúde. Coronavírus. Rio de Janeiro. Brasil

\section{THE CONTRIBUTION OF MEDICAL GEOGRAPHY AND HEALTH IN THE COVID-19 PANDEMIC}

\begin{abstract}
Health Geography isa a discipline that makes use of geographical concepts to work with the determinants of health and disease and the influences of social, environmental, economic and cultural aspects on the health os a specific population. The text brings a reflection on the importance of the Geography of Health during the pandemic of the new corona vírus, specifically in the city of Rio de Janeiro, Brazil. We consider that the entire pandemic process in Brazil was marked by denialism regarding disease and science, a fact that aggravated the situation of contaminated and dead people due to the lack of eficiente public policies that stimulated preventive measures that minimized the spread of the disease.
\end{abstract}

A reflection on the city of Rio de Janeiro

Keywords: Health Geography. Coronavirus. Rio de Janeiro. Brazil

\footnotetext{
${ }^{1}$ Mestre em Psicossociologia de Comunid. E Ecologia Social pela Universidade Federal do Rio de Janeiro. Pesquisador da Universidade do Estado do Rio de Janeiro.
} 


\section{INTRODUÇÃO}

O novo coronavírus começou a se manifestar em Wuhan, na China, sendo os primeiros casos da doença diagnosticados no final do ano de 2019. Rapidamente o vírus se espalhou pela Europa e chegou ao Brasil. A Organização Mundial da Saúde (OMS, 2020) declarou, em 30 de janeiro de 2020, que o surto da doença causada pelo novo coronavírus (COVID-19) constitui uma Emergência de Saúde Pública de Importância Internacional - o mais alto nível de alerta da Organização, conforme previsto no Regulamento Sanitário Internacional. Em 11 de março de 2020, a COVID-19 foi caracterizada pela OMS (2020) como uma pandemia. Do mês de março a agosto somaram o total de 24.011.502 casos confirmados no mundo, e 3.717.156, o Brasil com 117.665 casos de mortes em todo o país (OMS, 2020).

A doença é caracterizada por sintomas como febre, tosse, sintomas gripais, dor de garganta, diarreia, cansaço, perda do olfato e paladar e em casos mais graves, falta de ar e fibrose pulmonar, além do acometimento de outros órgãos e sintomas (FIOCRUZ, 2020a). A transmissão é feita através do contato com secreção contaminada, como gotículas de saliva, catarro, tosse ou espirro. As medidas de prevenção adotada são: lavar as mãos com água e sabão, uso de álcool, distanciamento social e uso de máscaras (FIOCRUZ, 2020b).

Com um quadro político instável e caótico o Brasil chegou a passar por três ministros da saúde no período de março a julho, quando então assumiu um ministro interino, coronel do exército, sem conhecimento técnico e permanece no cargo até o momento. O presidente do Brasil, Jair Bolsonaro, assumiu uma postura negacionista desde o início da pandemia, minimizando as consequências que esta doença poderia tomar e inclusive a qualificando como “gripezinha”, se recusava a usar máscaras, nega a ciência, questiona a Organização Mundial da Saúde e a Organização das Nações Unidas e apoia e indica um medicamento para uso precoce, que não têm eficácia cientifica comprovada e que é recusado pela maioria dos médicos.

Diante do quadro político tivemos uma polarização em relação as medidas de segurança, como o distanciamento social, uso de máscaras, álcool, e também, em relação a própria existência/gravidade da doença, o que acarretou em um número expressivo de casos e de mortes, chegando, o Brasil, a ser o epicentro da doença, juntos aos EUA, país esse que adotou uma postura parecida em relação a polarização e politização da doença.

Um ponto positivo e que salvou muitas vidas é que no Brasil, desde 1988 podemos contar com um sistema de saúde público, o Sistema Único de Saúde (SUS). O SUS é a tradução prática do princípio constitucional da saúde como direito de todos e dever do Estado (PAIM, 2009). A assistência é oferecida em um conjunto articulado de ações preventivas, curativas e de alta 
complexidade. Sua implementação avançou e estimulou, por exemplo, a descentralização do acesso aos serviços básicos de saúde.

O SUS tem a sua base de assistência organizada de acordo com o território, sendo os serviços oferecidos com base nas necessidades e realidades de cada população que habita determinado território. O primeiro contato do paciente com o sistema de saúde acontece nos postos de atenção primária ou atenção básica da saúde. Na cidade do Rio de Janeiro a atenção básica foi dividida por dez áreas programáticas, seguindo a lógica organizacional do SUS. É com base na territorialização do SUS e na divisão da assistência por áreas programáticas que propomos aqui uma intervenção da Geografia da Saúde na pandemia do novo Coronavírus, na cidade do Rio de Janeiro.

O texto é uma reflexão composto por essa introdução, uma secção sobre a Geografia da Saúde, que traz um breve histórico do conceito e também, de forma preliminar a organização territorial do SUS, uma secção que apresenta a proposta de intervenção e contribuição da Geografia da Saúde para a pandemia do COVID 19 na cidade do Rio de Janeiro, finalizando com as considerações finais.

\section{GEOGRAFIA DA SAÚDE E TERRITORIALIZAÇÂO DO SUS}

Embora os termos "Geografia Médica" e "Geografia da Saúde" sejam empregados para se referir aos estudos que fazem uso dos conceitos geográficos para trabalhar temas relacionados à saúde, existe uma diferença entre eles; Geografia Médica trata das doenças e suas causas relacionadas aos conceitos e condições geográficas e, a Geografia da saúde da ênfase ao suporte dos serviços oferecidos pela comunidade, "com evidência para o impacto dos cuidados de saúde na saúde e o bem estar da população" (SANTANA, 2014, p. 32).

Hipócrates foi um percursor na geografia médica ao associar o estudo das doenças com os lugares e o ambiente, e, primeira contribuição para a geografia médica é o tratado da escola de Hipócrates "Os ares, as águas e os lugares" que trazia estudos das doenças relativos aos fenômenos geofísicos, no final do século V a.C (SANTANA, 2014). Outros estudos surgiram nos séculos XVIII, XIX e XX voltados para a relação das doenças infecciosas e o ambiente (SANTANA, 2014).

O que ocorreu foi uma evolução no conceito, enquanto a Geografia Médica é caracterizada pela elaboração de mapas que representem a espacialização das diversas doenças, sem se aprofundar em analisar as causas das doenças, a Geografia da Saúde se aprofunda nas análises sobre saúde e doença (JUNQUEIRA, 2009). As mudanças nos quadros políticos e 
sociais que ocorrem a partir do século XIX, exigiram mudanças no campo da Geografia Médica, como as análises sociais no processo do adoecimento e da saúde. Em 1976, em Moscou, nasce a Geografia da Saúde, que sofre influência de vários autores da geografia, como Milton Santos (JUNQUEIRA, 2009, p. 1).

Alguns temas vêm sendo abordados por geógrafos, como: planejamentos dos serviços de saúde, distribuição e difusão das doenças, conflitos entre setor público e privado, enriquecendo a disciplina de Geografia da Saúde, o qual o objetivo geral dessa disciplina, Geografia da Saúde é, "proporcionar conhecimentos que sirvam para entender as relações que se estabelecem entre os condicionantes de saúde e os resultados efetivos na saúde, bem-estar e desenvolvimentos dos territórios" (SANTANA, 2014, p. 5).

Atualmente a Geografia da Saúde trata de questões relacionadas as desigualdades de acesso aos serviços de saúde, tendo em vista que grande parte dos artigos publicados nesta área são relativos às desigualdades em saúde, sobre as políticas públicas de saúde, envelhecimento e o aumento nas opções de cuidado com a saúde, na pobreza, e na exclusão, e as consequências na saúde das pessoas (SANTANA, 2014).

Um dos conceitos centrais da Geografia é o território, que é também utilizado no campo da saúde, que segundo Gondim e Mokem (2006, p. 3) "estruturam- se por meio de horizontalidades que se constituem em uma rede de serviços que deve ser ofertada pelo Estado a todo e qualquer cidadão como direito de cidadania"

Para viabilizar seus princípios, o SUS redesenhou as bases territoriais de acesso à saúde para assegurar a universalidade, a integralidade do cuidado e a equidade da atenção (GONDIM et al. 2008). Neste contexto a territorialização em saúde se coloca como uma metodologia capaz de operar mudanças no modelo assistencial, desenhando novas configurações loco-regional, baseando-se no reconhecimento e esquadrinhamento do território segundo a lógica entre ambiente, condições de vida, situação de saúde e acesso às ações de saúde (TEIXEIRA; PAIM; VILASBOAS, 1998).

Algumas ideias e movimentos contribuíram para o nascimento do SUS, o movimento que mais influenciou foi o movimento pela reforma sanitária que nasceu na década de 70, dentro de um contexto contra a ditadura e de um momento de intensas mudanças em busca da democracia. Nesta época a assistência à saúde pública era restrita a trabalhadores que contribuíam para a previdência Social, excluindo grande parte da população. O movimento entre outras questões tinha como objetivo mudanças no quadro de assistência à saúde. As propostas da reforma sanitária para a saúde eram: a saúde como direito de todo cidadão, no qual, as ações da saúde deveriam garantir o acesso da população às ações de cunho preventivo 


\section{ARTIGO}

INOVAÇÃo

e curativo; e a descentralização da gestão administrativa e financeira.

A intervenção de natureza estrutural feita pelo sistema de saúde no país com a criação do SUS e a descentralização da saúde dividiu o modelo de assistência em atenção primária, que priorizam a prevenção de doenças e a promoção da saúde que são oferecidos nos postos de atenção primária da saúde, atenção secundária que são entendidos como serviços especializados na medicina, como por exemplo, consultas com especialistas, serviços fisioterapia, psicoterapia entre outros e o nível terciário que se refere aos hospitais e serviços mais complexos de saúde (PAIM, 2009).

Os princípios doutrinários do SUS são: a) universalidade nos serviços, segundo o qual, a saúde é direito de todos e dever do estado, independente de vínculos empregatícios todos passam a ter direito à saúde; b) integralidade na assistência, as pessoas tem direito de serem atendidas na íntegra de suas necessidades, em um conjunto articulado de ações preventivas, curativas e de alta complexidade; c) equidade, tem relação direta com os conceitos de igualdade e justiça, busca-se com este princípio reconhecer as diferenças nas condições de vida e saúde e nas necessidades das pessoas.

A atenção primária, também chamada de atenção básica na saúde, pode ser entendida como o espaço onde acontece o primeiro contato do paciente com o sistema de saúde e onde se trata alguns adoecimentos sem grandes tecnologias evitando que a doença se agrave, e com isso, que necessitem de assistência médica especializada que utilizem grandes tecnologias, além de ser o espaço que se promova saúde e se previna doenças. O SUS desenvolve uma das propostas de atenção primária da saúde mais abrangentes, por meio do Programa de Saúde da Família - PSF (PAIM, 2009).

O termo atenção primária foi mencionado pela primeira vez pelo relatório de Dawson, em 1920 no Reino Unido, com objetivo de organizar os serviços de atenção à saúde por nível de complexidade e por uma base geográfica definida, assim este relatório serviu como base para orientar a organização dos serviços de saúde britânico e de outros países do mundo (LAVRAS, 2011).

No Brasil, o passo para a criação dos postos de atenção básica na saúde ou Programa Saúde da Família (PSF) foi dado a partir da criação do Departamento de Atenção Básica, estruturando a assistência e dividindo por áreas programáticas da saúde, possibilitando especializar e conhecer a realidade da situação de saúde das localidades específicas (GONDIM, 2008).

A atenção básica, na cidade do Rio de Janeiro, é dividida por dez áreas programáticas, (ver figura 1). A divisão das áreas é feita pela localização e proximidade dos bairros, seguindo 
INOVAÇÃo

a lógica do SUS, que tem como objetivo visualizar as condições de vida das pessoas e assim oferecer serviços de prevenção e promoção da saúde de acordo com a necessidade dos residentes de cada área.

Conforme o mapeamento e a ordenação de áreas para o planejamento de políticas públicas de promoção à saúde no Município do Rio de Janeiro (PERJ, 2018), as áreas programáticas da saúde na cidade são:

AP 1: Benfica, Caju, Catumbi, Centro, Cidade Nova, Estácio, Gamboa, Mangueira, Paquetá, Rio Comprido, Santa Teresa, Santo Cristo, São Cristóvão, Saúde e Vasco da Gama;

AP 2.1: Botafogo, Catete, Copacabana, Cosme Velho, Flamengo, Gávea, Glória, Humaitá, Ipanema, Jardim Botânico, Lagoa, Laranjeiras, Leblon, Leme, Rocinha, São Conrado, Urca e Vidigal;

AP 2.2: Alto da Boa Vista, Andaraí, Grajaú, Maracanã, Praça da Bandeira, Tijuca e Vila Isabel;

AP 3.1: Bonsucesso, Brás de Pina, Complexo do Alemão, Cordovil, Ilha do Governador, Jardim América, Manguinhos, Maré, Olaria, Parada de Lucas, Penha Circular, Penha, Ramos e Vigário Geral;

AP 3.2: Abolição, Água Santa, Cachambi, Del Castilho, Encantado, Engenho da Rainha, Engenho de Dentro, Engenho Novo, Higienópolis, Inhaúma, Jacaré, Jacarezinho, Lins de Vasconcelos, Maria da Graça, Méier, Piedade, Pilares, Riachuelo, Rocha, Sampaio, São Francisco Xavier, Todos os Santos e Tomás Coelho;

AP 3.3: Acari, Anchieta, Barros Filho, Bento Ribeiro, Campinho, Cascadura, Cavalcanti, Coelho Neto Barra de Guaratiba, Campo Grande, Cosmos, Guaratiba, Inhoaíba, Santíssimo, Senador Vasconcelos e Pedra de Guaratiba, Colégio, Costa Barros, Engenheiro Leal, Guadalupe, Honório Gurgel, Irajá, Madureira, Marechal Hermes, Oswaldo Cruz, Parque Anchieta, Parque Columbia, Pavuna, Quintino Bocaiuva, Ricardo de Albuquerque, Rocha Miranda, Turiaçu, Vaz Lobo, Vicente de Carvalho, Vila da Penha, Vila Kosmos e Vista Alegre.

AP 4.0: Barra da Tijuca, Camorim, Cidade de Deus, Grumari, Itanhangá, Jacarepaguá, Joá, Recreio dos Bandeirantes, Vargem Grande e Vargem Pequena;

AP 5.1: Bangu, Campo dos Afonsos, Deodoro, Jardim Sulacap, Magalhães Bastos, Padre Miguel, Realengo, Senador Camará e Vila Militar;

AP 5.2: Barra de Guaratiba, Campo Grande, Cosmos, Guaratiba, Inhoaíba, Santíssimo, Senador Vasconcelos e Pedra de Guaratiba;

AP 5.3: Paciência, Santa Cruz e Sepetiba. 
Figura 1 - Áreas programáticas de saúde na cidade do Rio de Janeiro/RJ

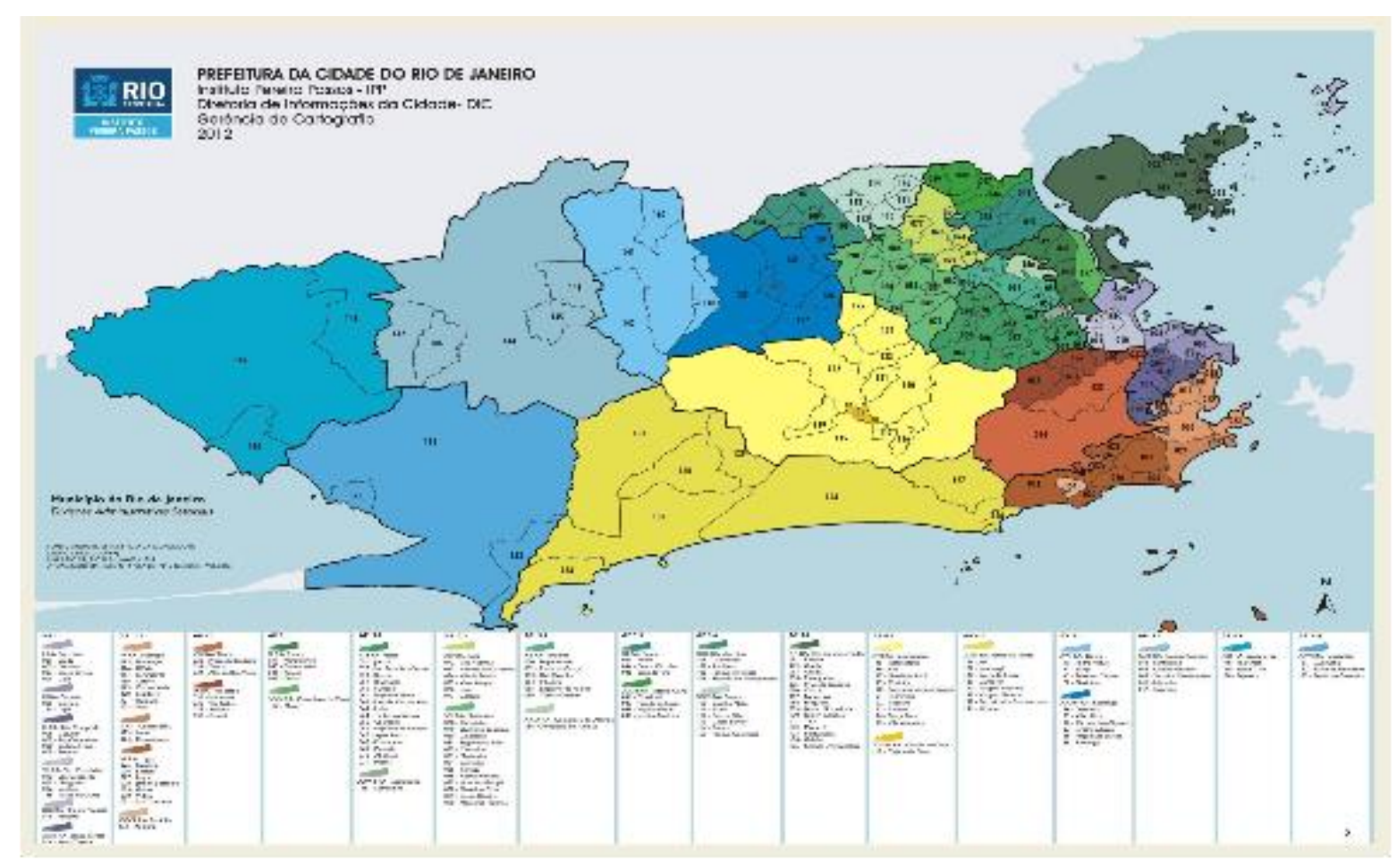

Fonte: Mapa obtido no Plano Municipal de Saúde, Prefeitura Municipal do Rio de Janeiro/RJ (2018)

\section{CONTRIBUiÇÕES DA GEOGRAFIA DA SAÚdE NA PANDEMIA DO CORONAVIRUS NA CIDADE DO RIO DE JANEIRO}

A cidade do Rio de Janeiro possui 6,32 milhões de habitantes, segundo dados do IBGE (2019). Até o dia 26 de agosto de 2020 o estado teve 216.675 casos confirmados e 15.700 mortes pelo novo coronavírus (GERJ, 2020); sendo o primeiro caso confirmado em 5 de março pela fundação Oswaldo Cruz (FUCHS, 2020). No dia 17 de março o prefeito do Rio de Janeiro, o bispo Marcelo Crivella, apresentou algumas ações para conter a pandemia, entre elas medidas que diminuam a circulação e aglomeração de pessoas em transportes públicos, suspensão das aulas nas escolas, fechamento de pontos turísticos da cidade, fechamento do comércio, entre outras medidas (PRJ, 2020). Em relação à assistência à saúde contamos com as orientações da vigilância sanitária, consultas telefônicas oferecidas pelo SUS e a nível do governo do estado, a montagem dos hospitais de campanha, compra de respiradores e contratação de mais profissionais da saúde para atuarem no nível terciário da saúde (PRJ, 2020).

Alguns escândalos de corrupção marcaram esta fase envolvendo o nome do atual governador do estado do Rio de Janeiro, Wilson Witzel no superfaturamento em compras dos respiradores para os hospitais de campanha (BRÍGIDO, 2020), mas nenhuma ação ou investimento na atenção básica da saúde foi feita pelo governo, em nenhuma das esferas 
(Municipal, Estadual, Federal) apenas ações isoladas que partiram de universidades, ONGS, grupos de profissionais etc. $\mathrm{O}$ investimento em ações de prevenção na atenção básica requer financiamento muito baixo e pode ser muito eficaz, principalmente para as populações em estado de vulnerabilidade socioeconômica.

A divisão da atenção básica em áreas programáticas permite visualizar as condições de determinada população e lança estratégias para conter a disseminação das doenças, pois a forma como a COVID-19 se comporta depende das condições de saneamento, habitação e infraestrutura, além de outros aspectos, como extensão territorial por exemplo. A Geografia da Saúde é de extrema relevância, pois a pandemia é também um problema geográfico e existe uma necessidade de georreferenciar, de avaliar a espacialidade dos casos confirmados, suspeitos, descartados, curados e as vidas perdidas. Estratégias de prevenção da contaminação do vírus podem ser realizadas de acordo com as realidades de cada população.

O Brasil é um país que apresenta uma desigualdade social e econômica acentuada e algumas medidas recomendadas pela OMS são desafiadoras para algumas famílias como por exemplo, a medida do distanciamento social, pois existem casos de pessoas que dividem um cômodo com mais de 5 familiares, e, as orientações de higiene das mãos e das roupas, existem bairros e comunidades que ficam semanas sem água, por isso a importância de informações para definir estratégias de acordo com as diversas realidades.

O quadro abaixo mostra um exemplo de como a Geografia da Saúde pode contribuir no mapeamento do perfil de uma população através de algumas informações que podem ser uteis para mapear os casos e os riscos de contaminação em cada área programática, as condições que a população tem de cumprir com determinadas medidas preventivas e quais as orientações e estratégias para conter a contaminação na região.

Quadro 1 - Exemplo de aplicação da Geografia da Saúde

\begin{tabular}{|l|}
\hline Área programática X \\
\hline Número de habitantes \\
\hline Número de casos confirmados \\
\hline População Idosa \\
\hline População com doenças pré-existentes \\
\hline Número de habitantes na residência \\
\hline Hábitos e Vícios \\
\hline Contaminados na família \\
\hline Quem trabalha/ Quem sai de casa \\
\hline Renda total da família \\
\hline
\end{tabular}

Fonte: elaborada pela própria autora, 2020. 
É um trabalho multidisciplinar, que une as estratégias e os dados da Geografia da Saúde, ao trabalho dos Agentes Comunitários de Saúde, Enfermeiros, Médicos e toda a equipe da atenção básica, podendo contribuir muito para o controle da pandemia.

\section{CONSIDERAÇÕES FINAIS}

A Geografia da Saúde tem potencial para contribuir com o controle da pandemia do CVID 19 por trabalhar com mapeamento de populações de riscos de doenças, e, de condições de vida das pessoas. A divisão da atenção básica em áreas programáticas facilita esse trabalho de mapeamento, contribuindo para a elaboração de estratégias de contenção da contaminação da doença por área definidas, considerando as diversas realidades de vida das pessoas que ali vivem.

No Brasil, a ausência de políticas públicas e de medidas efetivas de prevenção da doença fez com que o país chegasse a um número expressivo de mortes e de contaminação. No Rio de Janeiro, a baixa qualidade e efetividade das ações municipais e estaduais contribuíram para uma disseminação rápida do COVID 19 pela cidade.

O COVID é também, sem dúvida, um evento geográfico passando pelo processo de infecção que é definido pelo espaço que duas ou mais pessoas ocupam no ato da contaminação, ou seja, elas só se contaminam se estiverem ocupando um mesmo espaço e na disseminação mundial. As ações de medidas de contenção que se utilizam da divisão territorial do SUS e das áreas programáticas da saúde, se mostram eficazes para a elaboração de estratégias de contenção da doença. Se o método tivesse sido corretamente aplicado na cidade do Rio de Janeiro, principalmente desde o início da pandemia, os números de casos poderiam ter sido reduzidos, mas medidas que demandam de pouco investimento do dinheiro público parecem não ser tão atrativas para quem governa. 


\section{REFERÊNCIAS}

BRÍGIDO, Carolina. Witzel é incluído em inquérito sobre fraude em compra de respiradores. Extra, Glogo, 16 maio 2020. Disponível em: https://extra.globo.com/noticias/rio/witzelincluido-em-inquerito-sobre-fraude-em-compra-de-respiradores-rv1-1-24430625.html

CIDADE DO RIO DE JANEIRO. BRASIL. Ordenação de área para o planejamento. 2018. Disponível em:

https://mail.camara.rj.gov.br/APL/Legislativos/contlei.nsf/a99e317a9cfec383032568620071f 5d2/cdd6a33fa14df524832578300076df48/\$FILE/ATT53C5O.pdf/ANEXO\%20V.pdf

CIDADE DO RIO DE JANEIRO. BRASIL. Coronavírus: veja aqui as medidas adotadas pela Prefeitura do Rio para conter a pandemia na cidade. 24 mar. 2020. Disponível em: $\mathrm{http}$ ///prefeitura.rio/saude/coronavirus-veja-aqui-as-medidas-adotadas-pela-prefeitura-do-riopara-conter-a-pandemia-na-cidade/

FUCHS, Antônio. Covid-19: Instituto Nacional de Infectologia divulga boletim médico. 18 mar. 2020. Disponível em: https://portal.fiocruz.br/noticia/covid-19-instituto-nacional-deinfectologia-divulga-boletim-medico

FUNDAÇÂO OSWALDO CRUZ - FIOCRUZ. Como o coronavírus é transmitido?. 2020b. Disponível em: https://portal.fiocruz.br/pergunta/como-o-coronavirus-e-transmitido

FUNDAÇÂO OSWALDO CRUZ - FIOCRUZ. Tratamento do Coronavirus. 2020a. Disponível em: https://portal.fiocruz.br/pergunta/como-o-coronavirus-e-transmitido

GONDIM, G. M. M.; MONKEN, M.; ROJAS, L. I.; BARCELLOS, C.; PEITER, P.; NAVARRO, M. B. M. A.; GRACIE, R. O território da saúde: a organização do sistema de saúde e a territorialização. Rio de Janeiro: Fiocruz, 2008.

GONDIM, Grácia Maria de Miranda; MONKEN, Maurício. Territorialização em Saúde. Dicionário Educação da Profissional em Saúde, Fiocruz, 2006. Disponível em: http://www.epsjv.fiocruz.br/upload/ArtCient/25.pdf

GOVERNO DO ESTADO DO RIO DE JANEIRO -GERJ. Boletim Coronavírus (26/08): 15.700 óbitos e 216.675 casos confirmados no RJ. 26 ago. 2020. Disponível em: https://coronavirus.rj.gov.br/boletim/boletim-coronavirus-26-08-15-700-obitos-e-216-675casos-confirmados-no-rj/

IBGE. IBGE divulga as estimativa da população dos municípios para 2019. 28 ago. 2019. Disponível em: https://agenciadenoticias.ibge.gov.br/agencia-sala-de-imprensa/2013-agenciade-noticias/releases/25278-ibge-divulga-as-estimativas-da-populacao-dos-municipios-para2019

JUNQUEIRA. R. D. Geografia médica e geografia da saúde. Hygeia: Revista Brasileira de Geografia Médica e da Saúde, [S.1.], v. 5, n.8, p 1-10, out., 2009.

LAVRAS, Carmen. Atenção primária à saúde e a organização de redes regionais de atenção à saúde no Brasil. Saúde Soc., São Paulo, v. 20, n. 4, p. 867-874, dez. 2011. https://doi.org/10.1590/S0104-12902011000400005. 


\section{ORGANIZAÇÃO MUNDIAL DA SAÚDE. OMS afirma que COVID-19 é agora} caracterizada como pandemia. 2020. Disponível em:

https://www.paho.org/bra/index.php?option=com_content\&view=article\&id=6120:omsafirma-que-covid-19-e-agora-caracterizada-como-pandemia\& Itemid=812

PAIM, J. S. O que é o SUS. Rio de Janeiro: Editora FIOCRUZ, 2009.

PAIM, J. S. Reforma Sanitária Brasileira: contribuição para a compreensão e crítica. Rio de Janeiro: Editora FIOCRUZ, 2008.

SANTANA. P. Introdução a geografia da saúde: território, saúde e bem-estar. Coimbra, PT: Edição Empresa da Universidade de Coimbra, 2014.

TEIXEIRA, Carmem Fontes; PAIM, Jairnilson Silva; VILASBOAS, Ana Luiza. SUS, modelos de assistência e vigilância da saúde. Inf. Epidemiol. Sus, Brasília, v. 7, n. 2, p. 7-28, jun. 1998. http://dx.doi.org/10.5123/S0104-16731998000200002. 